Poster (E06)

Gestational Trophoblastic Neoplasia

https://doi.org/10.3802/jgo.2021.32.S1.E06

\section{Genomic profiles of gestational choriocarcinoma for diagnosis and predicting prognosis}

\section{Seongeun Bak, Youn Jin Choi*}

The Catholic University of Korea, Seoul St. Mary's Hospital, Seoul, Korea (yunno@catholic.ac.kr)

Objective: Gestational choriocarcinoma (GC) is one of gestational trophoblastic diseases (GTDs), and a unique cancer that originates in pregnant tissues. GCs are known to be preceded by complete hydatidiform mole (CHM) $(50 \%)$, previous abortions (25\%), or normal/ectopic pregnancies (25\%). CHM is considered a preneoplastic condition of GC, and invasive mole (IM) has neoplastic characteristics such as tissue invasion. However, what triggers CHM/IM to progress to $\mathrm{GC}$ is not figured out. Genomic alterations of GCs are not well studied because of the rare availability of tumor tissues. The lack of genome-wide alteration data on GTDs, led us to analyze GC genomes by whole exome sequencing, copy number alteration, copy-neutral loss-ofheterozygosity (CN-LOH) profiles in this study.

Methods: Whole-exome sequencing (20 GCs) and/or singlenucleotide polymorphism microarray (29 GCs) were performed. We analyzed CN-LOH in 29 GCs.

Results: Most GCs (25/29) harboring recurrent copy number alterations (CNAs) and gains on 1q21.1-q44 were significantly associated with poor prognosis. We detected five driver mutations in the GCs, most of which were chromatin remodeling gene (ARID1A, SMARCD1, and EP300) mutations but not in common cancer genes such as TP53 and KRAS.

Conclusion: Our data indicate that GCs have unique profiles of CN-LOHs, mutations and CNAs that together differentiate GCs from non-GCs. Practically, CN-LOH and CNA profiles are useful for the molecular diagnosis of GC and the selection of GC patients with poor prognosis for more intensive treatments, respectively.

Poster (E07)

Endometrial Hyperplasia, Endometrial Intra-epithelial Neoplasia, and Endometrial Cancer

https://doi.org/10.3802/jgo.2021.32.S1.E07

\section{Impact of surgical approach on survival and perioperative outcomes in women with stage II endometrial cancer}

Xian Li, ${ }^{1}$ Gladys Shuk Tak Kwok, ${ }^{2}$ Mandy Man Yee Chu, ${ }^{2}$ Siew Fei Ngu, ${ }^{2}$ Karen Kar Loen Chan, ${ }^{2}$ Hextan Yuen Sheung Ngan, ${ }^{3}$ Keedon Wong, ${ }^{2} \mathrm{Ka}$ Yu Tse ${ }^{3, *}$

'The University of Hong Kong - Shenzhen Hospital, Shenzhen, China

${ }^{2}$ The University of Hong Kong - Queen Mary Hospital, Hong Kong

${ }^{3}$ The University of Hong Kong, Hong Kong (tseky22@gmail.com)

Objective: To compare the survival and perioperative outcomes between minimally-invasive surgery (MIS) and open surgery in stage II endometrial cancer patients.

Methods: This was a retrospective study involving 205 patients with stage II endometrial cancer in a single institution between 2002-2018. Patients were stratified to either MIS or open surgery groups, and their pathological and clinical data were reviewed. Results: One hundred fourteen patients had open surgery (55.6\%) while 91 patients had MIS (44.4\%) including conventional laparoscopic $(70.3 \%)$, robot-assisted $(22.0 \%)$ and laparoscopic-assisted vaginal hysterectomy $(7.7 \%)$. The 5 - and 10-year overall survival rate was $94.5 \%$ and $93.4 \%$ for the MIS group, while $89.4 \%$ and $85.8 \%$ for the open surgery group $(p=0.219$ and $p=0.127)$. The 5 - and 10-year progression-free survival rate was $91.2 \%$ and $90.1 \%$ for the MIS group, while $86.8 \%$ and $81.5 \%$ for the open surgery group, without statistical significance, $\mathrm{p}=0.395$ and $\mathrm{p}=0.372$. The uterine size $(5.7 \pm 2.3$ vs. $8.0 \pm 4.0$ weeks; $\mathrm{p}=0.007)$ and tumor size $(2.5 \pm 1.7$ vs. $4.5 \pm 3.3$ $\mathrm{cm} ; \mathrm{p}=0.001$ ) were significantly smaller in the MIS group than in the open group. MIS was also associated with less blood loss (198.6 \pm 4.3 vs. $684.8 \pm 614.0 \mathrm{~mL}$; $\mathrm{p}=0.001)$, fewer wound complications ( $1.1 \%$ vs. $5.6 \%$; $=0.034)$, and shorter hospital stay ( 3 vs. 5 days; $p=0.031$ ) compared to open surgery. However, there is no significant difference in operation duration in the two groups ( $224 \pm 97$ vs. $227 \pm 94$ minutes; $p=0.554$ ).

Conclusion: The oncological outcomes and perioperative complication rates of patients undergoing MIS were not inferior to open surgery for stage II endometrial carcinoma.

\section{Poster (E08)}

Endometrial Hyperplasia, Endometrial Intra-epithelial Neoplasia, and Endometrial Cancer

https://doi.org/10.3802/jgo.2021.32.S1.E08

\section{Incorporation of protein biomarkers to the ProMisE molecular classifier for further prognostication in endometrial cancer}

Se Ik Kim, Hyunji Lim, Cheol Lee, Jae-Weon Kim, Maria Lee Seoul National University College of Medicine, Seoul, Korea (marialeemd@gmail.com)

Objective: The Proactive Molecular Risk Classifier for Endometrial Cancer (ProMisE) is a clinically applicable prognostication method 